\title{
Neutralino spin measurement with ATLAS detector at LHC
}

\author{
M. Biglietti ${ }^{1,2}$, I. Borjanovic ${ }^{3}$, G. Carlino ${ }^{2}$, F. Conventi ${ }^{1,2}$, E. Gorini ${ }^{3,4}$,

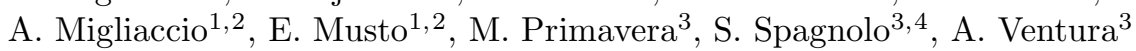 \\ 1 Dipartimento di Fisica - Università di Napoli "Federico II", Napoli, Italy \\ 2 Sezione INFN, Napoli, Italy \\ 3 Sezione INFN, Lecce, Italy \\ 4 Dipartimento di Fisica - Università del Salento, Lecce, Italy
}

\section{Introduction}

Supersymmetry (SUSY) is one of the leading candidates for physics beyond the Standard model (SM). Experimental SUSY studies are often performed in a minimal Supegravity inspired SUSY breaking framework, commonly referred to as mSUGRA. Within mSUGRA framework masses, mixings and decays of all SUSY and Higgs particles are determined in terms of four input parameters and a sign: the common mass $m_{0}$ of scalar particles at the grand unification scale, the common fermion mass $m_{1 / 2}$, the common trilinear coupling $A_{0}$, the ratio of the Higgs vacuum expectation values $\tan \beta$ and the sign of the supersymmetric Higgs mass parameter $\mu$. Once a signal of a physics beyond the Standard Model is seen at LHC, it will be fundamental to measure properties of new particles, like spin, in order to prove that they are indeed supersymmetric partners. The present work, described in detail in [2], is based on the analysis method proposed in [3] for the measurement of sparticle spins in cascade decays. Phenomenological studies $[4,5]$ show that this method allows the discrimination of different hypotheses for spin assignment, and can therefore e.g. be used for the discrimination of SUSY from Universal Extra Dimensions model which can mimick low energy SUSY at hadron colliders. In this report two selected points inside stau-coannihilation and bulk regions of the allowed mSUGRA parameter space are considered. Fast simulation [6] of the ATLAS detector was performed in order to investigate the feasibility of supersymmetric particles' spin measurement.

\section{Spin Measurement}

The cascade decay of the $\tilde{q}_{L}$ to $\tilde{\chi}_{2}^{0}$ which further decays to slepton 


$$
\tilde{q}_{L} \rightarrow \tilde{\chi}_{2}^{0} q \rightarrow \tilde{l}^{ \pm} l^{\mp} q \rightarrow l^{+} l^{-} q \tilde{\chi}_{1}^{0},
$$

can be used for the supersymmetric particles' spin measurement [3]. In the following, the first emitted lepton (the one from $\tilde{\chi}_{2}^{0}$ decay) is called near, and the one from slepton decay is called far. The second lightest neutralino has spin $1 / 2$ and the angular distribution of its decay products is not isotropic. This leads to a charge asymmetry of the invariant mass distributions $m\left(q l^{\text {near }( \pm)}\right)$ [3]. The invariant mass of quark and far lepton $m\left(q l^{f a r}\right)$ also possesses some small charge asymmetry [5]. It is not always possible to distinguish experimentally near from far lepton, so charge asymmetry of invariant mass of quark and lepton $m(q l)$ must be measured including contributions from both near and far leptons. This effect only causes a small dilution in the measurement. A more important effect is due to the fact that experimentally quark jets can not be distinguished from anti-quark jets. In the left handed anti-squark cascade decay, the asymmetry in the corresponding $m(\bar{q} l)$ charge distributions is the same as the asymmetry in $m(q l)$ from $\tilde{q}_{L}$ decay, but with opposite sign [7]. Therefore if one had the same number of squarks and antisquarks produced, the asymmetry effect would completely cancel out. However, at a proton-proton collider like the LHC, more squarks than anti-squarks will be produced, leading to a significant lepton charge asymmetry.

\section{SUSY Benchmark Points}

In the stau-coannihilation region (SU1 point) the lightest stau slepton $\tilde{\tau}_{1}$ is slightly heavier than the $\tilde{\chi}_{1}^{0}$ and acceptable relic density of cold Dark Matter is obtained through enhanced stau-neutralino coannihilation processes. The SU1 point has parameters $m_{0}=70 \mathrm{GeV}, m_{1 / 2}=350 \mathrm{GeV}, A_{0}=0 \mathrm{GeV}, \tan \beta=$ $10, \operatorname{sgn} \mu=+$. The $\tilde{q} / \overline{\tilde{q}}$ production ratio is approximately 3.5 , correspondingly enhancing the observability of the charge asymmetry. Main characteristic of SU1 point is that the mass difference between $\tilde{\chi}_{2}^{0}$ and $\tilde{l}_{L}$ is small $\left(m\left(\tilde{\chi}_{2}^{0}\right)-\right.$ $\left.m\left(\tilde{l}_{L}\right) \approx 10 \mathrm{GeV}\right)$ : as a consequence the near lepton has low $p_{T}$ in the $\tilde{\chi}_{2}^{0} \rightarrow$ $\tilde{l}_{L} l$ decay. Similarly, the small mass difference between $\tilde{l}_{R}$ and $\tilde{\chi}_{1}^{0}\left(m\left(\tilde{l}_{R}\right)-\right.$ $\left.m\left(\tilde{\chi}_{1}^{0}\right) \approx 20 \mathrm{GeV}\right)$, implies low values for far lepton's $p_{T}$ in $\tilde{\chi}_{2}^{0} \rightarrow \tilde{l}_{R} l$ decays. As a consequence, near and far leptons are distinguishable.

The bulk region (SU3 point) is an other of the allowed regions of mSUGRA parameter space. An acceptable relic density of cold Dark Matter is obtained thanks to the lightest neutralino annihilation processes via t-channel slepton exchange. The SU3 point has parameters $m_{0}=100 \mathrm{GeV}, m_{1 / 2}=300 \mathrm{GeV}$, $A_{0}=-300 \mathrm{GeV}, \tan \beta=6, \operatorname{sgn} \mu=+$. The squarks/anti-squarks production ratio is 2.5. Differently from the SU1 point, the mass of the second lightest neutralino $\left(m\left(\tilde{\chi}_{2}^{0}\right)=219 \mathrm{GeV}\right)$ is smaller than $\tilde{l}_{L}$ mass $\left(m\left(\tilde{l}_{L}\right)=230 \mathrm{GeV}\right)$. As a consequence, decays of $\tilde{\chi}_{2}^{0}$ to left-handed sleptons are forbidden and only decays to right-handed sleptons are allowed in the $\tilde{q}_{L}$ cascade decay. It is therefore not possible to experimentally distinguish near from far lepton 
in the bulk point. For this reason, when studying asymmetries, the invariant mass of quark and lepton $m(q l)$ is reconstructed summing contributions from near and far leptons, $m\left(q l^{\text {near }}\right)$ and $m\left(q l^{f a r}\right)$.

Events were generated with HERWIG 6.505 [8] by using the ISAWIG [9] interface with mass spectra and decay rates of supersymmetric particles given by ISAJET 7.69 and ISAJET 7.64 [10] for SU1 and SU3 points, respectively. SUSY samples corresponding to integrated luminosities $L=100 \mathrm{fb}^{-1}$ for SU1 point and $L=30 \mathrm{fb}^{-1}$ for SU3 point were analysed. Fast simulation studies on the most relevant SM background $(t \bar{t}+\mathrm{N}$ partons, $W+\mathrm{N}$ partons, $Z+$ $\mathrm{N}$ partons, produced with Alpgen 2.0.5 [11]) have been also performed.

\section{Event Selection}

A typical final state signature of $\tilde{q}_{L}$ cascade decays considered is given by large missing transverse energy, two same flavor opposite sign (SFOS) leptons, high- $p_{T}$ jets from the left-handed squark decay and from the decay of squark/gluino produced with $\tilde{q}_{L}$. Both SM and SUSY processes can mimick the final state signature of $\tilde{q}_{L}$ decay chain described above. Background can be classified as irreducible or reducible, depending on whether the two SFOS leptons $\left(e^{ \pm} e^{\mp}, \mu^{ \pm} \mu^{\mp}\right)$ in the event are correlated or not. The number of events with two uncorrelated SFOS leptons should be equal to the number of events with two opposite flavor opposite sign (OFOS) leptons $\left(e^{ \pm} \mu^{\mp}\right)$ and the corresponding dilepton invariant mass distributions should be identical. Consequently, reducible background can be suppressed by applying SFOS-OFOS subtraction $\left(e^{ \pm} e^{\mp}+\mu^{ \pm} \mu^{\mp}-e^{ \pm} \mu^{\mp}\right)$ on invariant mass distributions. The main SM background is $t \bar{t}+$ jets production. Because of the underlying event, pile-up and detector effects, other SM processes like $W / Z+$ jets can be also considered as sources of background. The SM background can be significantly reduced with hard kinematic cuts on missing transverse energy, number of jets and $p_{T}$ of jets. The following preselection cuts were applied:

- missing transverse energy $E_{T}^{\text {miss }}>100 \mathrm{GeV}$,

- at least four jets with transverse momentum $p_{T}\left(j_{1}\right)>100 \mathrm{GeV}$ and $p_{T}\left(j_{2}, j_{3}, j_{4}\right)>50 \mathrm{GeV}$,

- exactly two SFOS leptons (with transverse momenta $p_{T}^{\text {lepton }}>6 \mathrm{GeV}$ for SU1 point, and $p_{T}^{\text {lepton }}>10 \mathrm{GeV}$ for SU3 point).

From the three detectable particles $l^{+}, l^{-}$and $q$ (hadronized to jet) the leptonlepton $m(l l)$ and lepton-lepton-jet $m(j l l)$ invariant masses are formed. Here $j$ is one of the two most energetic jets in the event. These distributions show kinematic edges that, once measured, can be used for further selection:

- $m(l l)<100 \mathrm{GeV}, m(j l l)<615 \mathrm{GeV}$ (for the SU1 point),

- $m(l l)<100 \mathrm{GeV}, m(j l l)<500 \mathrm{GeV}$ (for the SU3 point). 


\begin{tabular}{|c|c|c|c|c|}
\hline & $\begin{array}{c}\text { Efficiency } \\
(\text { SU1 })\end{array}$ & $\begin{array}{c}\text { S/B } \\
(\text { SU1 })\end{array}$ & $\begin{array}{c}\text { Efficiency } \\
(\text { SU3 })\end{array}$ & $\begin{array}{c}\text { S/B } \\
(\text { SU3 })\end{array}$ \\
\hline Signal & $(17.0 \pm 0.3) \%$ & $/$ & $(20.0 \pm 0.3) \%$ & $/$ \\
\hline SUSY Background & $(0.94 \pm 0.01) \%$ & 0.33 & $(0.75 \pm 0.01) \%$ & 1 \\
\hline$t \bar{t}$ & $(2.69 \pm 0.02) 10^{-4}$ & 0.18 & $(3.14 \pm 0.02) 10^{-4}$ & 0.9 \\
\hline$W$ & $(1.4 \pm 0.9) 10^{-5}$ & $\sim 16$ & $(0.4 \pm 0.4) 10^{-5}$ & $\sim 300$ \\
\hline$Z$ & $(1.1 \pm 0.3) 10^{-5}$ & $\sim 12$ & $(0.9 \pm 0.2) 10^{-5}$ & $\sim 100$ \\
\hline
\end{tabular}

Table 1. Efficiencies and S/B ratios for SUSY signal and background (SU1, SU3) and for the most relevant sources of SM background $(l=e, \mu)$.

Efficiencies of all cuts described are shown in Table 1 together with the signal/background ratios. After SFOS-OFOS subtraction the contribution of SUSY background to the finally selected sample is further reduced by a factor $\sim 2$, and SM processes with two uncorrelated leptons become compatible with zero. For the SU1 point, four lepton-jet invariant masses have been reconstructed: $m\left(j l^{\text {near }}\right)_{L}$ and $m\left(j l^{f a r}\right)_{L}$, formed with jet and near lepton or jet and far lepton, respectively, in the case of $\tilde{\chi}_{2}^{0} \rightarrow \tilde{l}_{L} l$ decay and $m\left(j l^{\text {near }}\right)_{R}$ and $m\left(j l^{f a r}\right)_{R}$ for the corresponding cases of $\tilde{\chi}_{2}^{0} \rightarrow \tilde{l}_{R} l$. Decays with $\tilde{l}_{L}$ or $\tilde{l}_{R}$ are distinguished depending on the value of the dilepton invariant mass: $m(l l)<57 \mathrm{GeV}$ for $\tilde{l}_{L}$ and $57 \mathrm{GeV}<m(l l)<100 \mathrm{GeV}$ for $\tilde{l}_{R}$. Near and far leptons in both decays are selected according to their transverse momenta: in decay with $\tilde{l}_{L}$, the near (far) lepton is the one with lower (higher) transverse momentum, and vice versa for the decay with $\tilde{l}_{R}$. For the SU3 point there is no possibility to experimentally distinguish near and far lepton so their contributions were summed.

\section{Results}

From $m(j l)$ distributions the charge asymmetries have been determined. OFOS entries have been statistically subtracted from SFOS entries before computing the asymmetries. Two independent methods have been applied in order to estimate the presence of a non-zero charge asymmetry:

a. a comparison of asymmetry plots with a costant zero function (hypothesis of symmetry); a non parametric $\chi^{2}$ test provides a confidence level $\mathrm{CL}_{\chi^{2}}$,

b. a Run Test method [12]. A confidence level $\mathrm{CL}_{R T}$ for the hypothesis of no asymmetry is suitably defined.

The probabilities of $\mathbf{a}$ and $\mathbf{b}$ provide a combined confidence level $\mathrm{CL}_{c o m b}$. In Fig. 1 charge asymmetries are reported for $m\left(j l^{\text {near }}\right)_{L}$ in SU1 point and for $m(j l)$ in SU3 point, together with the confidence levels obtained with the two methods separately and combined. A value of $\mathrm{CL}_{\text {comb }}$ well below $1 \%$ is obtained for the SU1 point with $100 \mathrm{fb}^{-1}$, while $30 \mathrm{fb}^{-1}$ are sufficient to get 

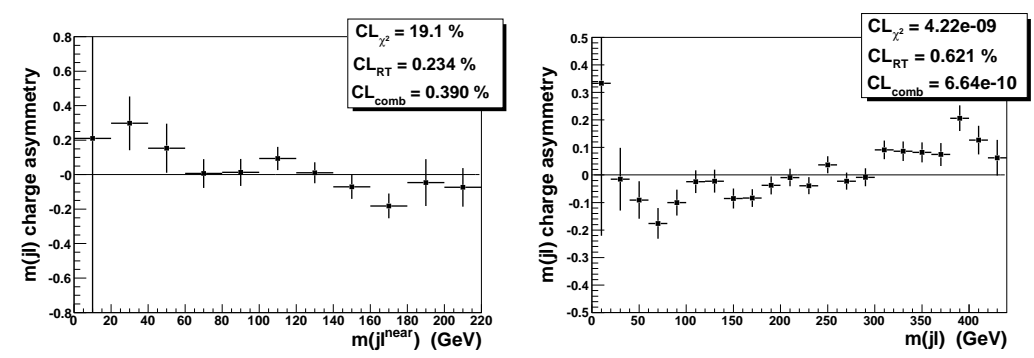

Fig. 1. Charge asymmetries for lepton-jet invariant masses after SFOS-OFOS subtraction. Left: using the near lepton from the chain involving $\tilde{l}_{L}$ in SU1 point. Right: using both near and far leptons in SU3 point.

a very low confidence level $\left(<10^{-9}\right)$ in the case of the SU3 point. Different sources of background and systematics have been evaluated [2] and show no significative effects on the capability of measuring the charge asymmetry. The whole analysis described so far has been repeated for different values of integrated luminosity: concerning SU1 point, the evidence with a $99 \%$ confidence level for a charge asymmetry would need an integrated luminosity of at least $100 \mathrm{fb}^{-1}$, while in SU3 point $m\left(j l^{ \pm}\right)$distributions can be excluded to be equal with a high probability already with less than $10 \mathrm{fb}^{-1}$.

\section{Acknowledgements}

This work has been performed within the ATLAS Collaboration, and we thank collaboration members for helpful discussions. The physics analysis framework and tools used in this work are the result of collaboration-wide efforts.

\section{References}

1. A. H. Chamseddine, R. Arnowitt and P. Nath, Phys. Rev. Lett. 49 (1982) 970.

2. M. Biglietti et al., ATLAS Note ATL-PHYS-PUB-2007-004 (2004).

3. A. J. Barr, Phys.Lett. B596 (2004) 205-212, [hep-ph/0405052].

4. A. Datta, K. Kong and K. T. Matchev Phys.Rev. D72 (2005) 096006, [hep$\mathrm{ph} / 0509246]$.

5. J. M. Smillie and B. R. Webber, JHEP 10 (2005) 069, [hep-ph/0507170].

6. E. Richter-Was, D. Froidevaux and L. Poggioli, ATLAS Internal Note ATLPHYS-98-131 (1998).

7. P. Richardson, JHEP 11 (2001) 029.

8. G. Corcella et al., JHEP 0101 (2001) 010, [hep-ph/0011363].

9. Program and its documentation are available from www.hep.phy.cam.ac.uk/ richardn/HERWIG/ISAWIG/

10. H. Baer et al., hep-ph/0001086. 
11. M.L. Mangano, M. Moretti, F. Piccinini, R. Pittau and A. Polosa, JHEP 0307 (2003) 001, [hep-ph/0206293].

12. A.G. Frodesen, O. Skjeggestad, H. Tofte, Probability and Statistics in Particle Physics, Bergen, Norway: Universitetsforlaget (1979) 